\title{
The spectral variability of HD 192639 and its implications for the star's wind structure
}

\author{
G. Rauw ${ }^{1, \star \star}$, N. D. Morrison ${ }^{2}$, J.-M. Vreux ${ }^{1}$, E. Gosset ${ }^{1, \star \star \star}$, and C. L. Mulliss ${ }^{2}$ \\ 1 Institut d'Astrophysique et de Géophysique, Université de Liège, 5 avenue de Cointe, 4000 Liège, Belgium \\ 2 Ritter Astrophysical Research Center, Toledo, OH 43606, USA
}

Received 7 August 2000 / Accepted 9 November 2000

\begin{abstract}
We report the analysis of an extensive set of spectroscopic data of the O(f) supergiant HD 192639. A Fourier analysis of our time-series reveals a recurrent variability with a "period" of roughly 4.8 days which is most prominent in the absorption components of the He II $\lambda 4686$ and $\mathrm{H} \alpha$ P-Cygni profiles. The same periodicity is also detected in the blue wing of several absorption lines (e.g. $\mathrm{H} \beta$ ). The variations of the absorption components correspond most probably to a cyclical modulation of the amount of stellar wind material along the line of sight towards the star. The 4.8-day period affects also the morphology of the double-peaked He II $\lambda 4686$ and H $\alpha$ emission components, although these emission components display also variations on other (mainly longer) time scales. The most likely explanation for the 4.8-day modulation is that this cycle reflects the stellar rotational period (or half this period). We find that the most important observational properties can be explained - at least qualitatively - by a corotating interaction region or a tilted confined corotating wind.
\end{abstract}

Key words. line: profiles - stars: early-type - stars: mass loss - stars: individual: HD 192639 - stars: variables: general

\section{Introduction}

Over the last decade, extensive observing campaigns have been organized to monitor the spectroscopic variability of early-type stars (e.g. Fullerton et al. 1996; Massa et al. 1995; Kaper et al. 1997, 1999). Most of the observed Ostars display line profile variability on time scales of a few days. Some well studied objects were found to exhibit cyclical variations in their stellar winds that are believed to be related to the rotational period of the underlying star. Such a rotational modulation is nowadays usually attributed to large scale structures in the wind that could be generated by photospheric processes affecting the mass outflow at the base of the wind (for a review see e.g. Fullerton 1999).

Understanding the physical mechanisms responsible for this modulation of the mass loss has become one of the primary goals of research in the field of hot stars. Nowadays, the most commonly invoked phenomena are magnetic fields and non-radial pulsations. An oblique magnetic rotator model has been used to explain the

\footnotetext{
Send offprint requests to: G. Rauw

* Based on observations collected at the Observatoire de Haute Provence, France and the Ritter Observatory, Toledo, USA.

$\star \star$ Postdoctoral Researcher FNRS, Belgium.

$\star \star \star$ Research Associate FNRS, Belgium.
}

cyclical variations of the peculiar $\mathrm{O} 7 \mathrm{~V}$ star $\theta^{1}$ Ori $\mathrm{C}$ (= HD 37022, Stahl et al. 1996), although we still lack any firm direct detection of the magnetic field of an O-star (Mathys 1999). On the other hand, non-radial pulsations have been detected in photospheric absorption lines of six O-stars so far (de Jong et al. 1999), but these pulsations have periods that are usually significantly shorter than the observed time scales of the stellar wind variations. Thus a variety of processes may be at work and there is a definite need for additional constraints on the stellar wind structure of early-type stars. This issue can best be addressed by extensive monitoring of selected stars.

In this context, Rauw \& Vreux (1998, hereafter Paper I) have started a detailed investigation of the O8 I f star HD 192639 in the Cyg OB1 association. In Paper I, we showed that the He II $\lambda 4686$ line displays a rather unusual double-peaked P-Cygni type profile that undergoes strong variability. The shape of this line indicates that the wind of HD 192639 is not spherically symmetric. In Paper I, we further showed that the variability of the He II $\lambda 4686$ profile is correlated to that of the $\mathrm{H} \beta$ absorption line. This led us to the conclusion that the variations seen in the spectrum of HD 192639 arise probably from a large scale structure in the stellar wind rather than from a photospheric phenomenon. Based on these preliminary results, we tentatively suggested a recurrence time of the variations of $\sim 5$ days. 
To further constrain the properties of the spectral variability of HD 192639, we have obtained new data and we have extended our analysis to the spectral region around the $\mathrm{H} \alpha$ emission line.

\section{Observations and data reduction}

The bulk of our new spectroscopic observations of HD 192639 were collected during two observing campaigns in September 1998 and August 1999 with the Aurélie spectrograph fed by the $1.52 \mathrm{~m}$ telescope of the Observatoire de Haute-Provence (OHP). In 1998 we used a 600 lines $/ \mathrm{mm}$ grating blazed at $5000 \AA$ providing a reciprocal dispersion of $16 \AA / \mathrm{mm}$. Two wavelength settings ranging from 4460 to $4880 \AA$ and 6360 to $6740 \AA$ were covered. The detector was a Thomson TH7832 linear array with a pixel size of $13 \mu \mathrm{m}$. A total of 25 blue and 18 red spectra were obtained over a ten night run. The mean exposure time was about $45 \mathrm{~min}$ and the mean $S / N$ ratio in the continuum was $\sim 200$ and $\sim 400$ in the blue and red wavelength ranges respectively. The spectral resolution as derived from the FWHM of the ThAr calibration lines is $\sim 0.6 \AA$. Whenever the atmospheric conditions allowed us to do so, we obtained a set of blue spectra at the beginning and at the end of each night in order to study shorter time scale variations.

The 1999 data set was obtained with a 300 lines $/ \mathrm{mm}$ grating blazed at $6000 \AA$ providing a reciprocal dispersion of $33 \AA / \mathrm{mm}$ over a wavelength range from 4100 to $4950 \AA$. The instrumental configuration used in 1999 yielded a spectral resolution of $\sim 1.2 \AA$. We obtained 14 spectra with a $S / N \simeq 200$ over a 15 night run. Typical exposure times were $30-45 \mathrm{~min}$.

All the OHP data were reduced in the standard way using the MIDAS software developed at ESO. The spectral domain between 6420 and $6640 \AA$ is affected by strong absorption bands of telluric water vapor. A template of the telluric spectrum was constructed by observing the rapidly rotating star HD 177724 (A0 Vp, $m_{V}=2.99$, $v_{e} \sin i=295 \mathrm{~km} \mathrm{~s}^{-1}$ Abt \& Morrell 1995) at very different airmasses. This template was then used for a first order correction of the telluric features. Finally, the spectra were normalized using properly chosen continuum windows.

Eight spectra of HD 192639 were also obtained with the echelle spectrograph attached to the 1-m RitcheyChrétien reflector of the Ritter Observatory (Toledo, USA). Five of these spectra were obtained concurrently with the September 1998 run at OHP as part of a multisite campaign. The instrument was equipped with a liquid nitrogen cooled CCD camera system supplied by Wright Instruments Ltd. The detector was a $1200 \times 800 \mathrm{EEV} \mathrm{CCD}$ chip with pixel dimensions of $22.5 \mu \mathrm{m} \times 22.5 \mu \mathrm{m}$. The resolving power was 26000 and the typical $S / N$ ratio was about 75 for a typical exposure time of one or two hours depending on the weather conditions. The data reduction used the Interactive Data Language (IDL) with a specific program written for Ritter Observatory data. The individual echelle orders of the Ritter data cover a rather narrow
Table 1. Summary of our observing runs. The violet, blue, red and $\mathrm{H} \alpha$ wavelength ranges stand respectively for $4100-4460 \AA$, 4460-4880 $\AA, 6360-6740 \AA$ (except $\mathrm{H} \alpha$ ) and 6560-6590

\begin{tabular}{lcccc}
\hline & violet & blue & red & $\mathrm{H} \alpha$ \\
\hline \hline$\Delta T^{\text {tot }}$ (days) & 1094.09 & 1094.09 & 9.07 & 36.91 \\
$N$ & 40 & 65 & 18 & 28 \\
$\overline{\Delta t}$ (days) & 0.081 & 0.098 & 0.052 & 0.069 \\
$\Delta T_{\text {run }}^{\max }($ days $)$ & 13.93 & 13.93 & 9.07 & 13.95 \\
$\Delta \nu_{\text {nat }}\left(\mathrm{d}^{-1}\right)$ & 0.001 & 0.001 & 0.110 & 0.027 \\
$\nu_{\max }\left(\mathrm{d}^{-1}\right)$ & 6.2 & 5.1 & 9.6 & 7.2 \\
$\nu_{\min }\left(\mathrm{d}^{-1}\right)$ & 0.072 & 0.072 & 0.110 & 0.072 \\
\hline
\end{tabular}

spectral range and we therefore used the lower resolution OHP spectra as templates to normalize the Ritter spectra.

In addition to these new observations, we have also included the 1996 and 1997 Aurélie data discussed in Paper I in our analysis. A summary of the characteristics of our various data sets is presented in Table 1. $\Delta T^{\text {tot }}$ gives the total time elapsed between our first and our last observation, while $N$ is the total number of observations. $\overline{\Delta t}$ provides the average time interval between consecutive exposures obtained during the same night. $\Delta T_{\text {run }}^{\max }$ yields the time spanned by the longest observing run in our data set. In light of the Fourier analysis in Sect. 3.1, we further list the typical width of a peak in the power spectrum $\Delta \nu_{\text {nat }}=1 / \Delta T^{\text {tot }}$. Due to the occurrence of bundles of aliases with nearly identical amplitude, the actual uncertainty on the peak position is larger than $\Delta \nu_{\text {nat }}$ for the blue and violet data set; typical uncertainties are of the order of $0.005 \mathrm{~d}^{-1}$. $\nu_{\max }=1 /(2 \overline{\Delta t})$ provides a rough indication of the highest frequencies that could safely be studied with the present time series. Finally, $\nu_{\min }=1 / \Delta T_{\text {run }}^{\max }$ yields the shortest frequency that could possibly be fully sampled within the longest data subset.

\section{Results}

To start, we have identified those lines in the spectrum of HD 192639 that display the most prominent variations. To this aim we have computed the temporal variance spectrum (TVS, Fullerton et al. 1996) of our entire data set. Although we rectified our spectra in a homogeneous way, small amplitude discrepancies in the normalization procedure cannot be ruled out and they might induce some artificial "variability". We have evaluated an upper limit on this apparent variability due to the normalization by computing the $T V S$ over the broad diffuse interstellar band at $4430 \AA$ and over the narrow DIB at $\lambda 6614$. In this way we find that the normalization errors should be limited to $T V S^{1 / 2} \leq 0.004^{1}$. A Fourier analysis of the artificial

\footnotetext{
${ }^{1}$ Due to the broad wings of the $\mathrm{H} \alpha$ emission line, the residuals of the telluric features and the rather narrow spectral range covered by the Ritter data, the normalization errors at $\mathrm{H} \alpha$ could be slightly larger.
} 

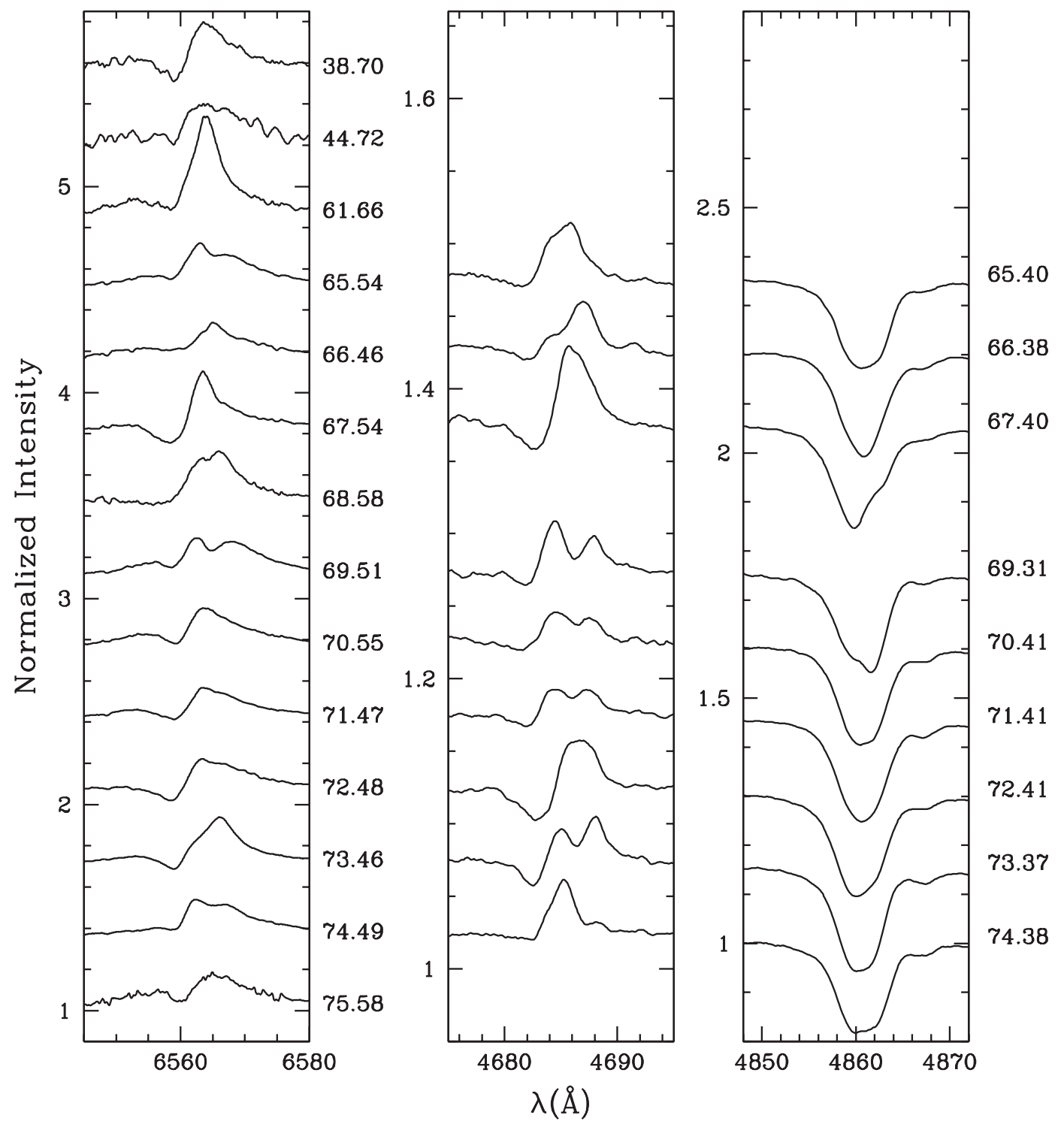

Fig. 1. Examples of the night to night variability of the $\mathrm{H} \alpha$ (left), He II $\lambda 4686$ (middle) and $\mathrm{H} \beta$ (right) line profiles in the normalized spectrum of HD 192639 as observed in September 1998 from OHP and Ritter Observatory. The various spectra are shifted by $0.35,0.05$ and 0.15 units respectively for the $\mathrm{H} \alpha$, He II $\lambda 4686$ and $\mathrm{H} \beta$ profiles. The labels yield the mean date of the observation in the format JD-2451000.0. Note the very different vertical scales of the different panels

variability of the interstellar features reveals that this effect mainly appears as a long-term trend.

As already discussed in Paper I, the most significant profile variations in the blue-violet wavelength range are found in the He II $\lambda 4200, \mathrm{H} \gamma, \mathrm{He}$ I $\lambda 4471$, C III $\lambda \lambda 4647$ 50 and $\mathrm{H} \beta$ absorption lines as well as in the He II $\lambda 4686$ P-Cygni type profile and, to some extent, the $\mathrm{N}$ III $\lambda \lambda$ 4634-41 emission line. To this list we can now add the He I $\lambda 6678$ absorption line and the $\mathrm{H} \alpha$ P-Cygni type profile in the red wavelength range. Our new data confirm the low amplitude of the variations in the He II $\lambda \lambda 4542$, 6406,6527 and $\lambda 6683$ absorption lines $\left(T V S^{1 / 2} \leq 0.007\right.$ for the blue line and $\leq 0.005$ for the red lines) and the lack of variations in the Si IV $\lambda \lambda 6668,6701$ emission lines $\left(T V S^{1 / 2} \leq 0.003\right)$.
The night to night profile variability of the $\mathrm{H} \alpha$, He II $\lambda 4686$ and $\mathrm{H} \beta$ lines in the spectrum of HD 192639 as observed in September 1998 is shown in Fig. 1. Although subtle changes on time scales of a few hours are certainly present, the most prominent variations occur over time scales of a few days.

The $\mathrm{H} \alpha$ line consists of a P-Cygni type profile overlying a broad emission component extending from 6530 to $6590 \AA$. The wings of the broad emission component exhibit very little variability (see Fig. 5), whereas the PCygni like core of the profile displays prominent variations. The absorption component varies between a well defined trough and a very discrete, hardly noticeable absorption. The emission component changes from a sharp singlepeaked normal P-Cygni emission to a double-peaked 
somewhat broader structure. The intensity of the emission peak varies between 1.16 and 1.28 times the intensity of the continuum. On one occasion (JD 2451061.66), we observe an increase of the peak intensity to 1.45 times the continuum.

The variations of the He II $\lambda 4686$ and $\mathrm{H} \beta$ lines are very reminiscent of those already described in Paper I. The He II $\lambda 4686$ line displays various kinds of P-Cygni profiles quite similar to what we observe for the $\mathrm{H} \alpha$ line, although the correspondence between the profiles of the two lines is certainly not perfect. Usually, the double-peaked structure of the emission component is more pronounced in the He II $\lambda 4686$ line. The core of the $\mathrm{H} \beta$ line displays strong variations and changes from a more or less symmetric profile to a strongly asymmetric shape. At the same time we notice that the width of the line is also variable; for instance the line appears slightly broader on JD 2451067.40 than on JD 2451066.38. To quantify this effect, we have measured the width of the $\mathrm{H} \beta$ line profile at a normalized intensity of 0.9 , i.e. at about half the line intensity (the FWHM cannot be used here since the depth of the line is itself slightly variable). Figure 2 illustrates the variation of this parameter and shows that the width increases by about $10 \%$ on $\sim$ JD 2451067.4 and JD 2451072.4. These width increases mainly result from a shift of the blue wing by about 30-40 $\mathrm{km} \mathrm{s}^{-1}$ towards shorter wavelengths. These events occur at epochs when the P-Cygni absorption components of the $\mathrm{H} \alpha$ and $\mathrm{He}$ II $\lambda 4686$ lines exhibit their strongest intensity and the strongest blueward extension. Conversely, when the strongest peak of the P-Cygni emission component reaches its maximum redward excursion, we notice a weakening of the red wing of the $\mathrm{H} \beta$ absorption line.

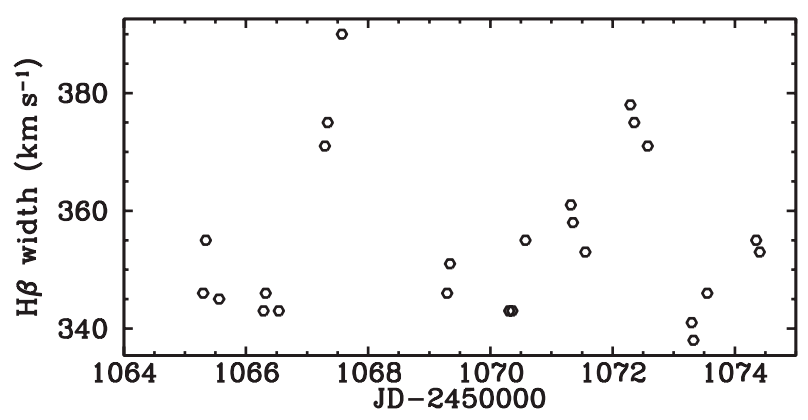

Fig. 2. Variations of the width of the $\mathrm{H} \beta$ absorption line at 0.9 times the intensity of the continuum

\subsection{Searching for periodic line profile variability}

Our time series contains data subsets from different epochs and different instrumentations. In order to improve the $S / N$ and to avoid spurious effects in the time series analysis due to the different spectral resolutions, the Ritter data were filtered using a running average filter to achieve an effective resolution roughly matching that of the 1998 OHP data. For the Fourier analyses, the spectra from the different subsets were rebinned onto a heliocentric frame of reference using a uniform wavelength step of $0.2 \AA$.

We have used the "generalized spectrogramme" Fourier method of Heck et al. (1985; see their Sect. 2.2) to derive the power spectrum of our spectroscopic time series $^{2}$. This method allows a proper estimation of the power spectrum in the case of an uneven sampling.

The resulting 2-D power spectrum of the He II $\lambda 4686$ line is displayed as a grey-scale representation in Fig. 3. The most outstanding feature of the power spectrum is a peak at $\nu_{1}=0.210 \pm 0.005 \mathrm{~d}^{-1}(P=4.76 \pm 0.10$ days $)$ with its aliases at $0.18,0.24,0.76,0.79$ and $0.82 \mathrm{~d}^{-1}$. Although this structure is visible over the entire profile, it is most prominent in the wavelength range $[4681.5,4684.5] \AA$ (i.e. over the velocity range $\left.[-270,-77] \mathrm{km} \mathrm{s}^{-1}\right)$. Figure 4 shows the 1-D power spectrum obtained by integrating the 2-D periodogram over the latter wavelength range as well as the same 1-D power spectrum "prewhitened" for $\nu_{1}$ (see below) and the spectral window up to $5.0 \mathrm{~d}^{-1}$. Above $5.0 \mathrm{~d}^{-1}$, the power spectrum is essentially featureless without any further prominent peak. In Fig. 4, we see that the aliasing of the $\nu_{1}$ frequency can account for all the significant peaks in the $1-\mathrm{D}$ power spectrum. To check this, we have prewhitened the data for the $\nu_{1}$ frequency. This means that at every wavelength-step (or radial velocitystep $v$ ) we have fitted an expression

$$
\begin{aligned}
F(v, t)= & c_{0}(v)+a_{1}(v) \sin \left(2 \pi \nu_{1}\left[t-t_{0}\right]\right) \\
& +b_{1}(v) \cos \left(2 \pi \nu_{1}\left[t-t_{0}\right]\right)
\end{aligned}
$$

to our time series. The sinusoidal variation of frequency $\nu_{1}$ was then subtracted from our data and the Fourier technique was applied to the resulting time series. The corresponding prewhitened power spectrum reveals no trace of any other significant peak in the $[4681.5,4684.5] \AA$ wavelength range (see Fig. 4).

The situation is a bit less clear for the red emission component of the profile. There is still a peak at $\nu_{1}$, but it no longer provides the dominating feature in the power spectrum over that part of the line profile. Prewhitening the data for $\nu_{1}$ according to Eq. (1) reveals two peaks at $\nu_{2}=0.086 \pm 0.010 \mathrm{~d}^{-1}$ and $\nu_{3}=0.38 \pm 0.20 \mathrm{~d}^{-1}$. The highest peak at $\nu_{2}$ is rather close to $\nu_{\min }=0.072 \mathrm{~d}^{-1}$ i.e. the shortest frequency that could possibly be fully sampled within our longest data subset. The significance of this peak is therefore highly uncertain. In the following we will refer to this feature as "long term variability". The bundle of peaks in the prewhitened power spectrum around $\nu_{3}$ is quite broad and asymmetric. We notice that the position of the $\nu_{3}$ feature is at least not inconsistent with the first harmonic of the $\nu_{1}$ frequency though one should not overinterpret this result.

\footnotetext{
2 To assure that our time-series analysis is not affected by systematic effects due to the rather heterogeneous data set, we have also performed Fourier analyses of the individual data subsets. The resulting power spectra are very similar to those obtained for the entire data set, though the peaks have of course different widths.
} 

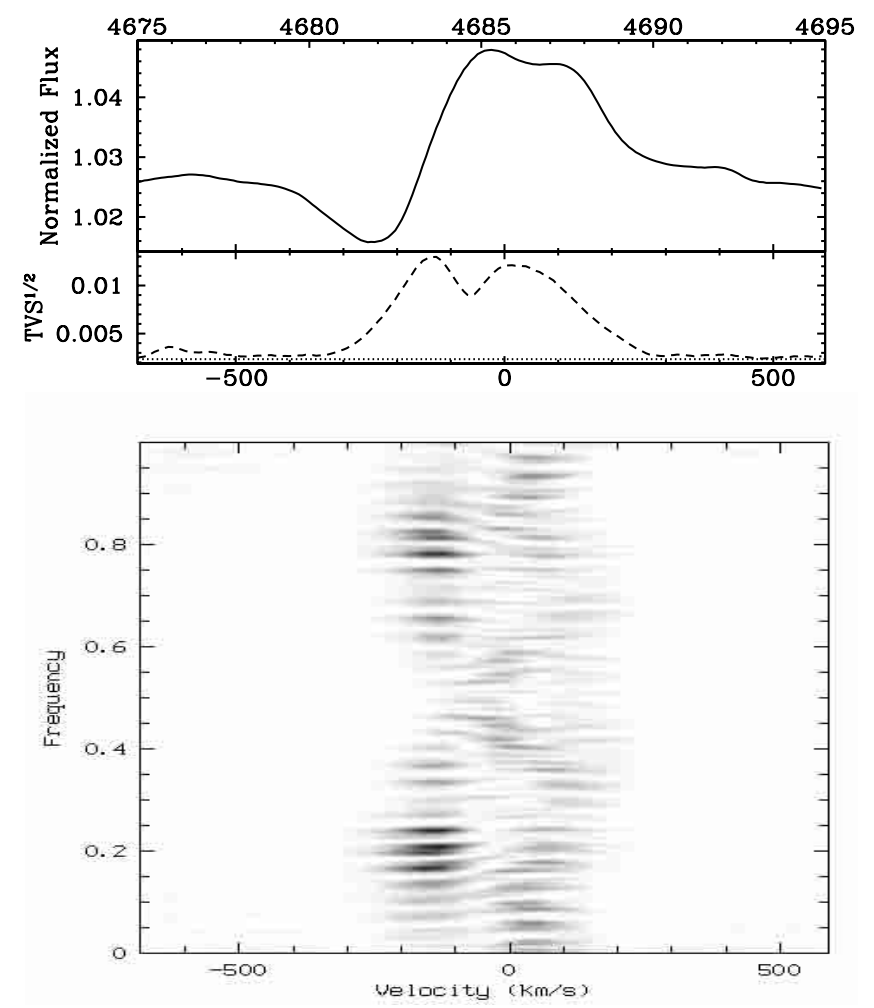

Fig. 3. Mean profile of the He II $\lambda 4686$ line in the spectrum of HD 192639 as observed with the Aurélie spectrograph at OHP from 1996 to 1999. The top axis of the upper panel indicates the wavelength whilst the bottom axis provides the corresponding radial velocity. The middle panel displays the square root of the temporal variance spectrum and the lower panel provides a grey-scale image of the power spectrum in the range $[0.0,1.0] \mathrm{d}^{-1}$ computed using the Fourier technique of Heck et al. (1985). The power spectrum reveals a family of peaks around $\nu_{1}=0.21 \mathrm{~d}^{-1}$

The power spectrum of the $\mathrm{H} \alpha$ line is shown in Fig. 5 . The temporal variance spectrum indicates that the variability is concentrated in the P-Cygni core of the line, whereas the extended wings of the line show no sign of variability. Though the natural width of the peak is of course much larger for the $\mathrm{H} \alpha$ data set (see Table 1) than for the He II $\lambda 4686$ data, one clearly sees that the highest peak in the blue part of the profile is again found near $\nu \sim \nu_{1}$ with its $1-\nu$ alias around $0.8 \mathrm{~d}^{-1}$. This peak dominates in the wavelength range $[6553,6562] \AA$. Figure 6 further demonstrates the lack of power at higher frequencies and the decreasing amplitude of the aliases. The situation in the red emission component is again more complex. The highest peaks are $0.97 \mathrm{~d}^{-1}$ and its alias at $0.03 \mathrm{~d}^{-1}$. We notice however that these peaks vanish if we omit the spectrum taken on JD 2451061.66, while the power spectrum over the blue part of the line profile remains unchanged. On the JD 2451061.66 spectrum HD 192639 displays an unusually strong $\mathrm{H} \alpha$ emission component (see Fig. 1). Again we will consider the 0.97 and $0.03 \mathrm{~d}^{-1}$ features as an indication of a, most probably non-periodic, long-term variability.
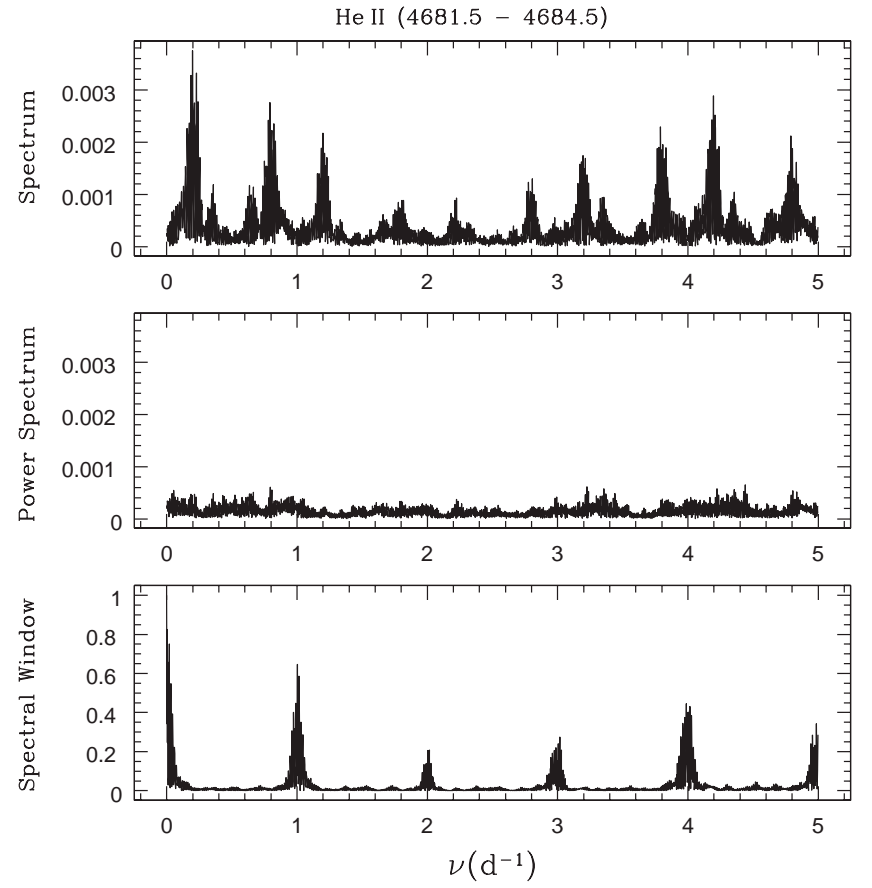

Fig. 4. Upper panel: power spectrum of the absorption component of the He II $\lambda 4686$ line profile integrated over the wavelength range 4681.5 to $4684.5 \AA$. Middle panel: power spectrum prewhitened for $\nu_{1}=0.21 \mathrm{~d}^{-1}$. Lower panel: power spectral window corresponding to our data set in the blue range

Finally, the power spectrum of the $\mathrm{H} \beta$ line (Fig. 7) reveals a clear dichotomy between the behaviour in the blue wing $\left(4856-4860 \AA\right.$, i.e. $\left.[-330,-80] \mathrm{km} \mathrm{s}^{-1}\right)$ compared to the core of the line and its red wing. In the blue wing, the highest peak is once more found at $\nu_{1}=0.21 \mathrm{~d}^{-1}$. On the other side, the highest peaks in the core of the line correspond to $0.02 \mathrm{~d}^{-1}$ and its aliases. Most of the absorption lines in the blue spectrum of HD 192639 display a power spectrum similar to that of the $\mathrm{H} \beta$ line. A summary of our Fourier analyses is given in Table 2. In most cases, we find the dominant peak in the blue wing of the line either at $\nu_{1}=0.21 \mathrm{~d}^{-1}$ or at the $0.18 \mathrm{~d}^{-1}$ alias. The power spectra of the red wing and the core of the absorption lines exhibit their highest peaks in the range $[0.01,0.02] \mathrm{d}^{-1}$ with the corresponding - nearly equally strong - aliases near $0.98 \mathrm{~d}^{-1}$. The low-frequency peaks probably reveal either the existence of a long-term trend in the spectrum of HD 192639 or the occurrence of an exceptional event in the present time series. Conversely, we find no peak at higher frequencies that could be indicative of a photospheric variability on shorter time scales (e.g. due to non-radial pulsations). We caution however that many of the absorption lines of HD 192639 might at least partially - be filled in by stellar wind emission. Moreover, our time series is not well suited to investigate variability on time scales of several hours as expected for non-radial pulsations.

In summary, our Fourier analysis reveals a roughly "periodic" behaviour especially in the blue-shifted absorption component of the $\mathrm{H} \alpha$ and $\mathrm{He}$ II $\lambda 4686$ P-Cygni 

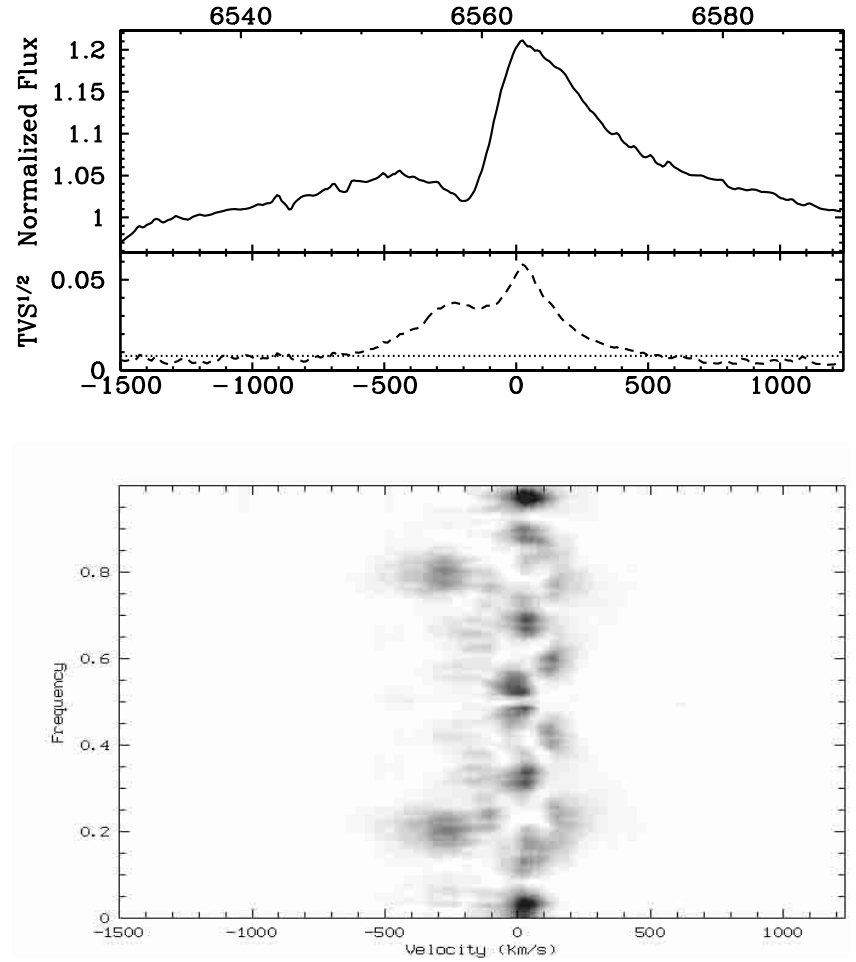

Fig. 5. Same as Fig. 3 but for the profile of the $\mathrm{H} \alpha$ line in the spectrum of HD 192639 as observed at OHP and at the Ritter Observatory in 1998. The power spectrum indicates a peak near $\nu \sim 0.2 \mathrm{~d}^{-1}$ and another one near $1.0 \mathrm{~d}^{-1}$ in the emission component. The latter feature is strongly dependent upon the inclusion of the JD 2451061.66 spectrum in the analysis (see text)
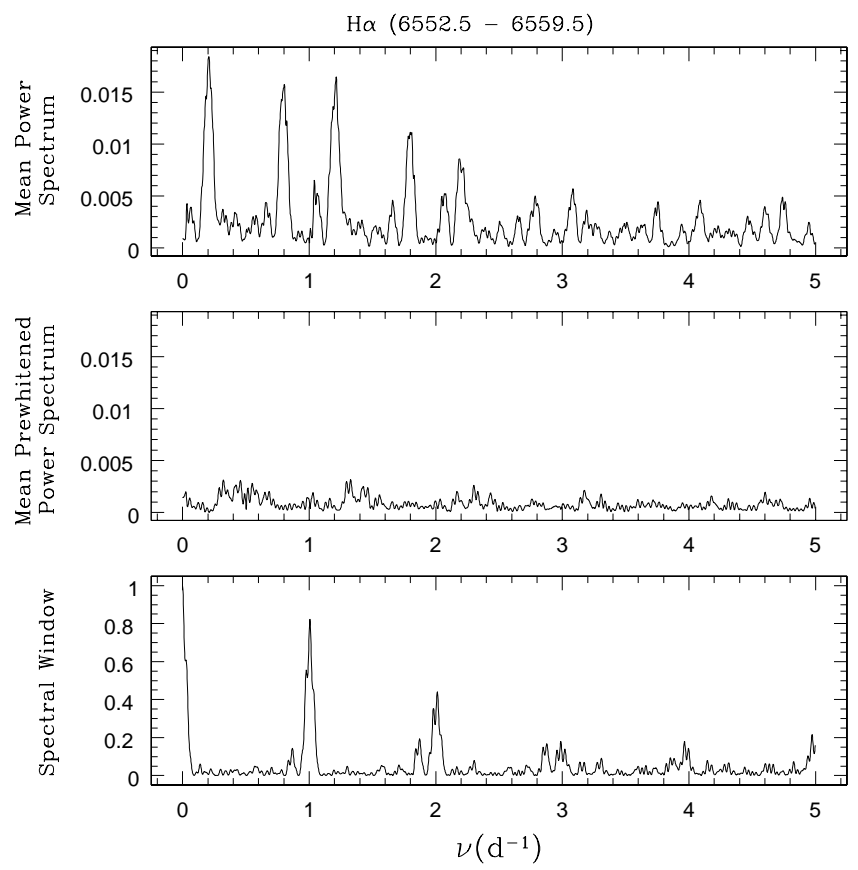

Fig. 6. Same as Fig. 4 but for the $\mathrm{H} \alpha$ line profile integrated over the wavelength range 6552.5 to $6559.5 \AA$

profiles on a recurrence time of 4.8 days. This result is in very good agreement with our previous suggestion
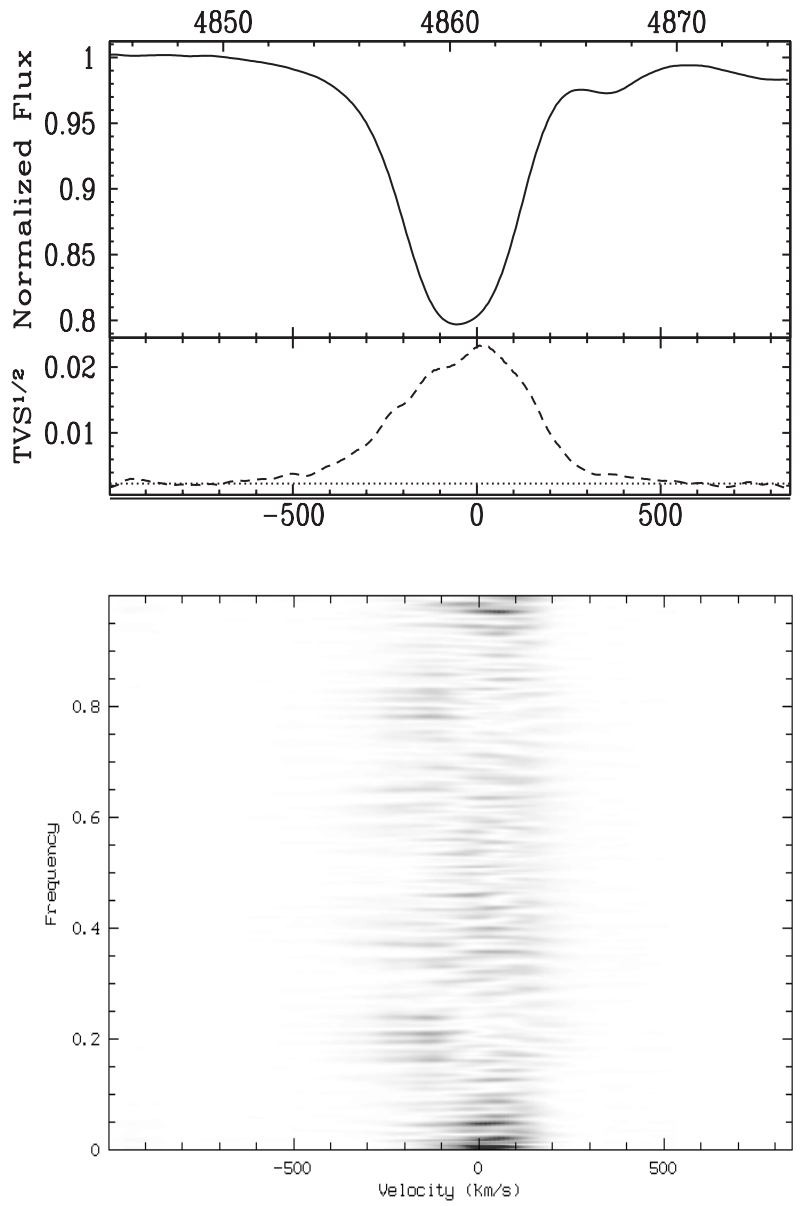

Fig. 7. Same as Fig. 3 but for the profile of the $\mathrm{H} \beta$ absorption line

(Rauw \& Vreux 1998). The periodogram of the emission component of the He II $\lambda 4686$ line reveals also some power near the first harmonic of this period, but the latter detection is not secure. We find also strong evidence for longterm variability in the red part of the line profiles, but the corresponding "periods" are longer than our individual observing runs and no consistent result is found for the different lines.

\subsection{The variations with the $\nu_{1}$ frequency}

In order to sample the complete 4.76-day cycle, we have binned the blue data set into twenty equal phase intervals, each containing at least two spectra. The resulting dynamical spectrum of the He II $\lambda 4686$ line is displayed in Fig. 8. This figure illustrates the modulation of the intensity of the absorption component of the P-Cygni profile as well as the changes in the relative intensity of the two peaks of the emission component.

In principle, we can use the information from our Fourier analysis to restore the pattern of variability associated with the $\nu_{1}$ frequency. To this aim, we have fitted 
Table 2. Summary of the Fourier analyses of our time series of spectra. Columns (2) and (3) list the frequency of the highest peak in the power spectrum respectively over the entire profile and in the blue wing. The flag given in Col. (4) refers to the data sets described in Table 1 . The $0.97 \mathrm{~d}^{-1}$ peak in the power spectrum of the $\mathrm{H} \alpha$ line is obtained including the "unusual" spectrum on JD 2451061.66 (see text)

\begin{tabular}{lccc}
\hline \multicolumn{1}{c}{ Line } & $\begin{array}{c}\text { Highest peak } \\
\text { entire profile }\end{array}$ & $\begin{array}{c}\text { Highest peak } \\
\text { blue wing }\end{array}$ & Data set \\
\hline He II $\lambda 4200$ & $1.00 \mathrm{~d}^{-1}$ & - & violet \\
H $\gamma$ & $\leq 0.005 \mathrm{~d}^{-1}$ & $\nu_{1}$ & violet \\
He I $\lambda 4471$ & $0.02 \mathrm{~d}^{-1}$ & $0.18 \mathrm{~d}^{-1}$ & blue \\
He II $\lambda 4542$ & $0.02 \mathrm{~d}^{-1}$ & - & blue \\
N III $\lambda 4640$ & $0.18 \mathrm{~d}^{-1}$ & $0.18 \mathrm{~d}^{-1}$ & blue \\
C III $\lambda \lambda 4647-50$ & $0.04 \mathrm{~d}^{-1}$ & $0.18 \mathrm{~d}^{-1}$ & blue \\
He II $\lambda 4686$ & $\nu_{1}$ & $\nu_{1}$ & blue \\
H $\beta$ & $0.02 \mathrm{~d}^{-1}$ & $\nu_{1}$ & blue \\
H $\alpha$ & $\left(0.97 \mathrm{~d}^{-1}\right)$ & $\nu_{1}$ & H $\alpha$ \\
He I $\lambda 6678$ & $0.20 \mathrm{~d}^{-1}$ & $0.20 \mathrm{~d}^{-1}$ & red \\
\hline
\end{tabular}

an expression

$$
\begin{aligned}
F(v, t)= & c_{0}(v)+\sum_{i=1}^{n}\left\{a_{i}(v) \sin \left(2 \pi \nu_{i}\left[t-t_{0}\right]\right)\right. \\
& \left.+b_{i}(v) \cos \left(2 \pi \nu_{i}\left[t-t_{0}\right]\right)\right\}
\end{aligned}
$$

to our time series at every velocity step. Equation (2) differs from Eq. (1) in the sense that we simultaneously fit up to three frequencies $\nu_{i}$. By this means, we are able to account for the specific sampling of the most prominent other frequencies that contribute to the power spectrum and to properly isolate the $\nu_{1}$ variations from the effects of the other trends that exist in the emission component of the P-Cygni profile. The amplitude $\sqrt{a_{1}(v)^{2}+b_{1}(v)^{2}}$ and the phase $\phi_{0}(v)=\arctan \left[b_{1}(v) / a_{1}(v)\right]$ of the $\nu_{1}$ sinewave are shown as a function of radial velocity $v$ in Fig. 8 . Figure 9 shows the reconstruction of the $\nu_{1}$ periodic modulation in Eq. (2) over the He II $\lambda 4686$ line.

We caution that the phase constant $\phi_{0}$ has physical meaning only if the period is clearly detected at the specific velocity. Since $\nu_{1}$ is not the dominant frequency in the emission component, one could expect a priori that $\phi_{0}$ might be slightly more uncertain there than in the absorption component. We have tested the restoration technique (Eq. (2)) with $n=1,2$ and 3. These calculations reveal that the overall behaviour of $\phi_{0}$ is quite robust over the entire velocity range where $\nu_{1}$ is clearly detected.

The phase diagram and the reconstruction technique reveal several interesting features:

- the phase of the modulation $\phi_{0}(v)$ remains roughly constant over the absorption component but changes dramatically over the emission component. The overall behaviour of $\phi_{0}(v)$ is independent of the number of additional frequencies included in the fit of Eq. (2);

- there is a local minimum in the amplitude of the sinusoidal variations near $v \sim-40 \mathrm{~km} \mathrm{~s}^{-1}$;
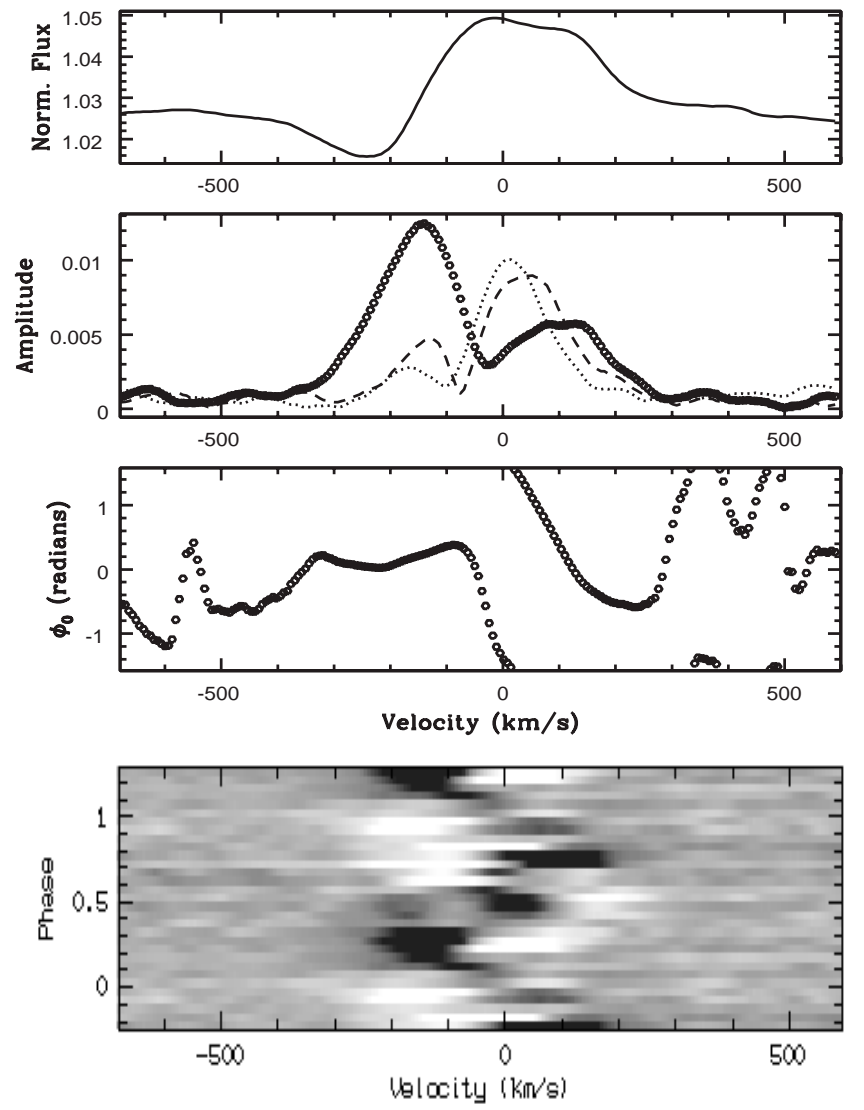

Fig. 8. Variability of the He II $\lambda 4686$ line as a function of phase assuming a period of 4.76 days. The three upper panels display the mean line profile, as well as the amplitude and the phase of a sine wave of frequency $\nu_{1}$ fitted to our data. The reference date for the phase was arbitrarily chosen on JD 2450000.00. The amplitude of the two other frequencies that were fitted simultaneously are shown for comparison: $0.086 \mathrm{~d}^{-1}$ (dotted line), $0.38 \mathrm{~d}^{-1}$ (long-dashed line). The greyscale image displays the ratio of the actual spectrum to the mean profile. The black pixels correspond to a ratio $\leq 0.99$, whereas the white pixels indicate a ratio $\geq 1.01$

- the intensity of the emission component seems to vary roughly in phase with the strength of the absorption component i.e. the phase-lag between maximum emission strength and maximum absorption is less than 0.08 cycle. At the phase of maximum emission, we observe a single-peaked, flat-topped emission component centred on a velocity of $v \sim+50 \mathrm{~km} \mathrm{~s}^{-1}$;

- conversely the visibility of the blue emission peak decreases when the strength of the absorption increases. In fact, near maximum absorption strength, the blue peak vanishes and the line displays a "normal" PCygni type profile.

We have applied the same techniques to the $\mathrm{H} \alpha$ data set but using only ten bins in the phase diagram to sample the complete 4.76-day period (since the phase-coverage is less complete for this data set). The resulting dynamical 

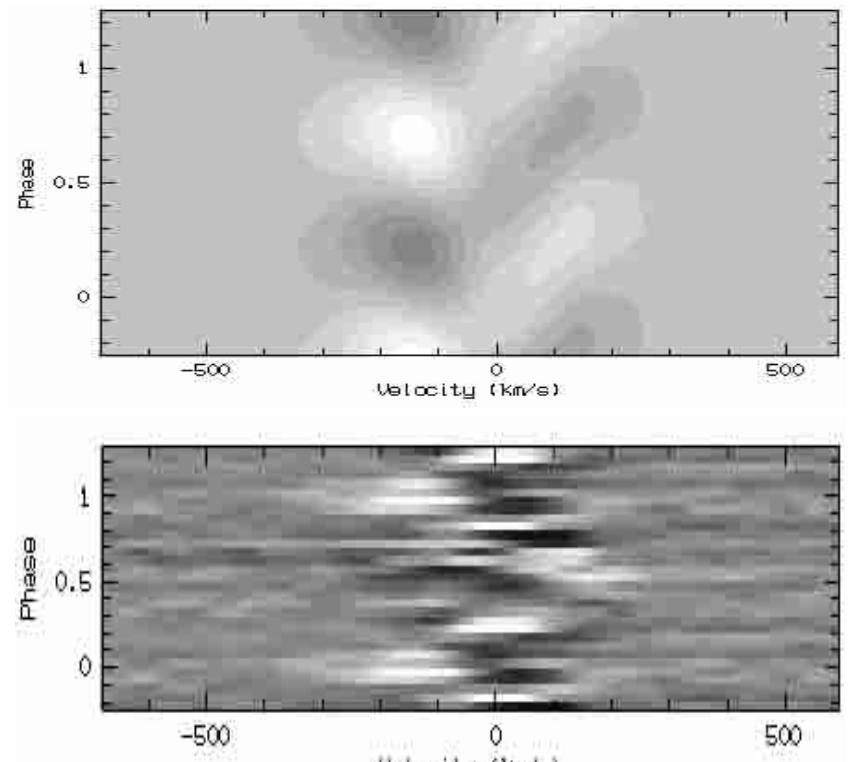

Velocity (kirvs)

Fig. 9. Upper panel: reconstruction of the modulation of the He II $\lambda 4686$ line as a function of phase assuming a period of 4.76 days. The amplitude and phase constant used in the reconstruction are obtained from Eq. (2) and are displayed in Fig. 8. The black pixels correspond to a deficit in the normalized intensity of more than $\geq 0.012$ compared to the mean profile, whereas the white pixels indicate an excess intensity of $\geq 0.012$. Lower panel: grey-scale image of the residuals (observed - calculated) as a function of phase. This image shows that the variations in the velocity range $[-300,-50] \mathrm{km} \mathrm{s}^{-1}$ are very well matched by our restoration of the $\nu_{1}$ frequency, whereas the variations in the emission component are heavily affected by the other frequencies

spectrum and reconstruction are shown in Figs. 10 and 11 respectively. We notice the following characteristics:

- contrary to the situation of the He II $\lambda 4686$ line, the phase constant $\phi_{0}(v)$ shows a steady decrease towards more negative velocities across the $\mathrm{H} \alpha$ absorption component;

- there are two local minima in the amplitude of the sinusoidal variations near $v \sim-25 \mathrm{~km} \mathrm{~s}^{-1}$ and $+100 \mathrm{~km} \mathrm{~s}^{-1}$;

- the variations of the intensity of the emission peak are less clearly related to those of the absorption component than in the case of the He II $\lambda 4686$ line.

We have measured the equivalent widths of the absorption components in the $\mathrm{H} \alpha$ and He II $\lambda 4686$ P-Cygni profiles. A Fourier analysis of the $E W$ s of the He II $\lambda 4686$ absorption yields the strongest peak at $0.2075 \mathrm{~d}^{-1}$, which is an alias of $\nu_{1}\left(\nu_{1}\right.$ itself being the second highest peak). The phase diagram of the $E W \mathrm{~s}$ is shown in Fig. 12. Although there is considerable scatter in this diagram, we notice that the $E W \mathrm{~s}$ of the absorption components of both lines undergo variations by more than a factor of three. The $E W$ s are larger between phase $\sim 0.1$ and $\sim 0.45$ and are in fact maximum around phase 0.3 . The duration and amplitude of the absorption enhancement reveal an increase of
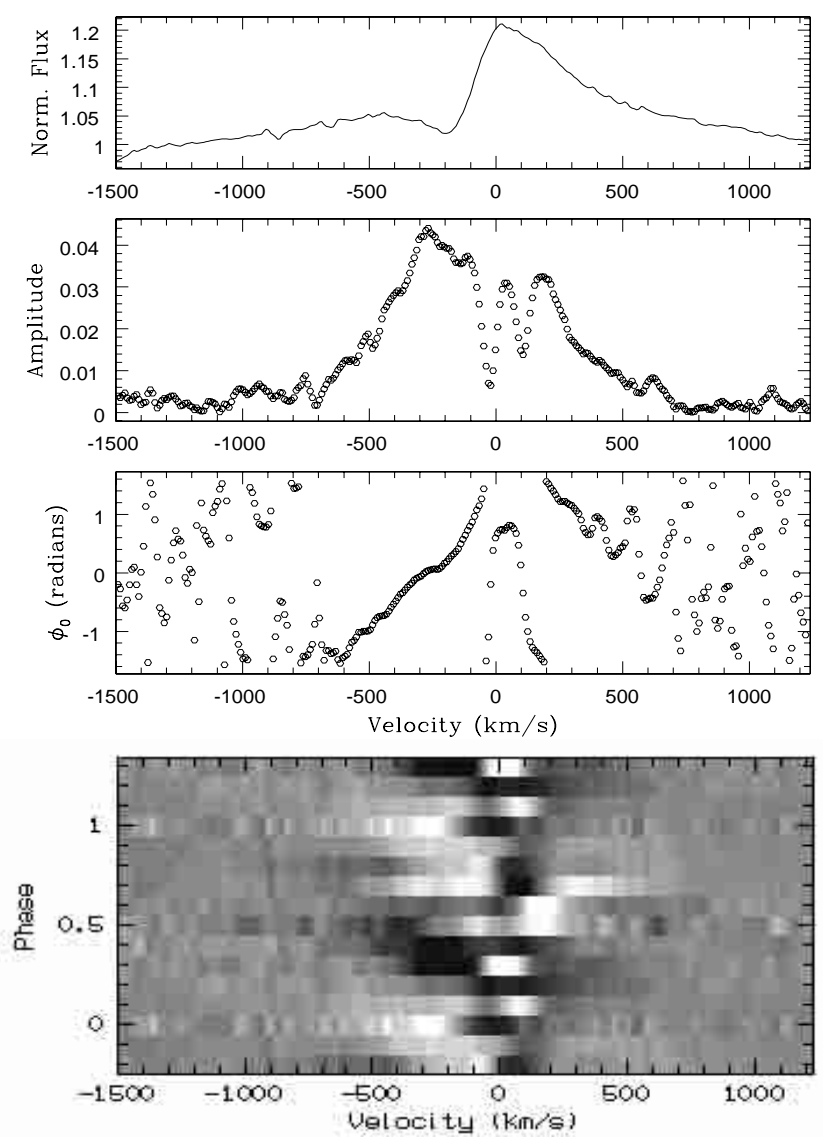

Fig. 10. Same as Fig. 8 but for the $\mathrm{H} \alpha$ line. The black pixels in the dynamical spectrum correspond to a ratio $\leq 0.96$, whereas the white pixels indicate a ratio $\geq 1.04$

the density and/or the opacity in a significant fraction of the wind column towards the stellar surface. There is no evidence for a significant phase-lag between the variations of the equivalent widths of the absorption components of the two lines.

The pattern of the variability of the $\mathrm{H} \beta$ line with the $\nu_{1}$ frequency is very similar to the one of the He II $\lambda 4686$ line. This similarity is not unexpected given the correlation between the variability of both lines found in Paper I. This result indicates that the variations in the blue wing of the $\mathrm{H} \beta$ line with $\nu_{1}$ are most probably due to a changing amount of wind absorption and emission rather than to a genuine variability of the underlying photospheric absorption.

\subsection{Radial velocities and equivalent widths of the absorption lines}

In Paper I, we concluded that binarity was not a likely explanation for the variability of the He II $\lambda 4686$ line observed in the spectrum of HD 192639. To check this conclusion, we have measured the radial velocities of the most prominent absorption lines on our new data by fitting Gaussian profiles. The results confirm the very small range of variability of the radial velocities already found in 

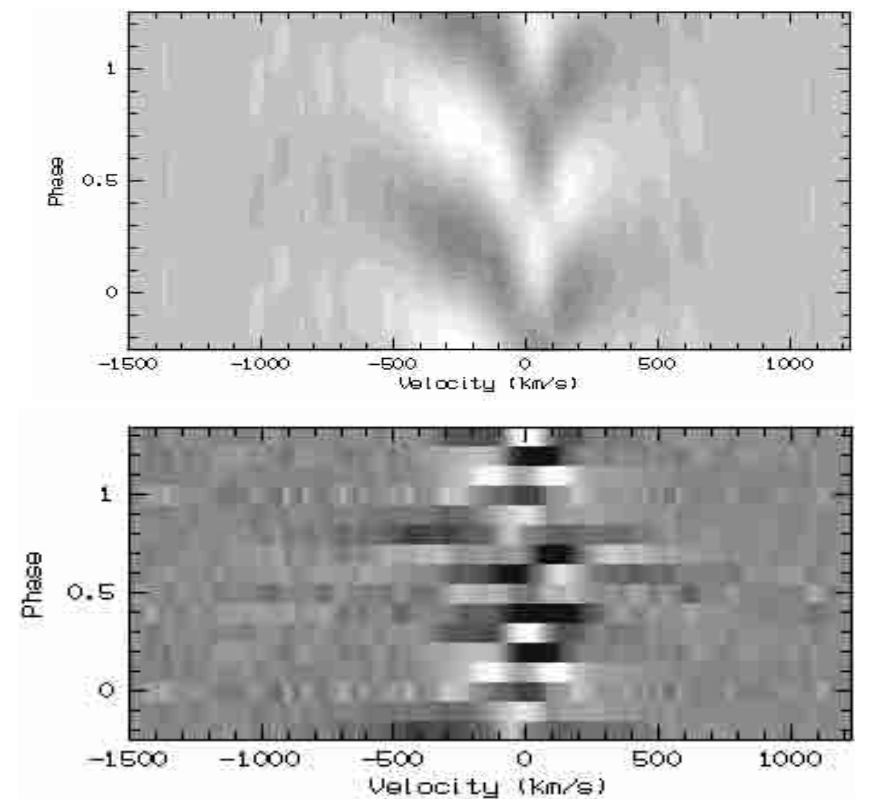

Fig. 11. Same as Fig. 9 but for the $\mathrm{H} \alpha$ line. The amplitude and phase constant of the $\nu_{1}$ modulation were obtained from Eq. (2) including a simultaneous fit of the $0.03 \mathrm{~d}^{-1}$ frequency. The black pixels in the dynamical spectrum correspond to a deficit of more than $\geq 0.044$, whereas the white pixels indicate an excess of $\geq 0.044$
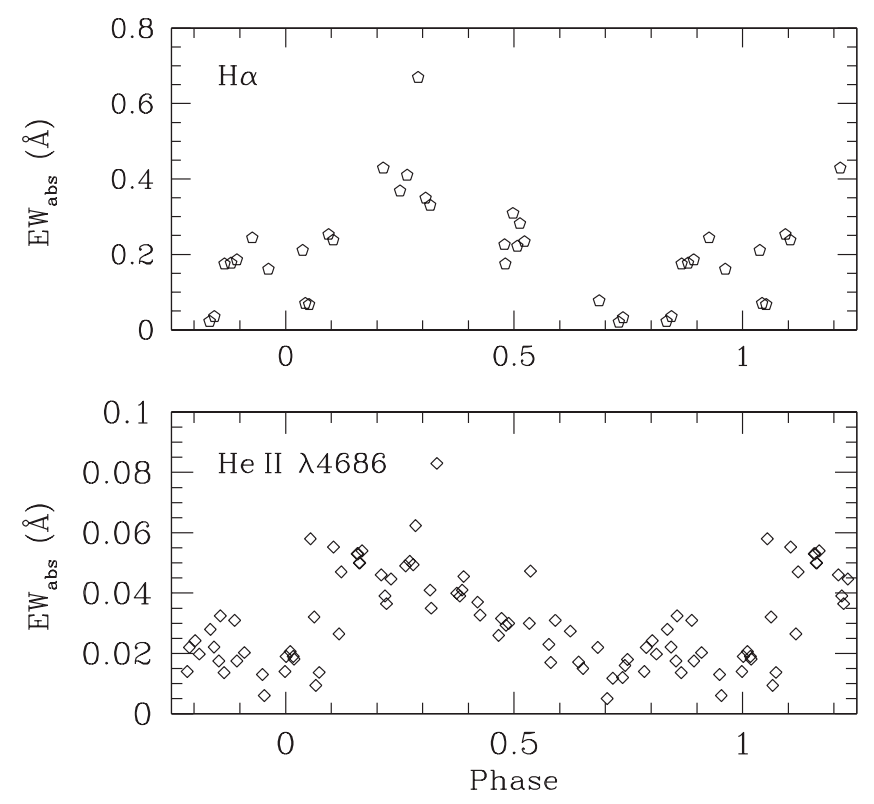

Fig. 12. Phase diagram of the $E W$ s of the absorption components of the $\mathrm{H} \alpha$ and He II $\lambda 4686$ lines assuming a period of 4.76 days. Phase 0.0 was arbitrarily chosen on JD 2450000.00

Paper I. For instance, for the He II $\lambda 4542$ line we can set an upper limit of $5 \mathrm{~km} \mathrm{~s}^{-1}$ on the semi-amplitude of any radial velocity variation in a putative single-lined spectroscopic binary.
We applied a Fourier analysis to the RVs of the individual absorption lines. It turns out that the RVs of different spectral lines yield quite different power spectra with the most prominent peak occurring usually at frequencies below $0.02 \mathrm{~d}^{-1}$. None of the power spectra displays a significant peak near $\nu_{1}$. Nevertheless, we have folded the RV data with the 4.76-day "period" determined above. The result is shown in Fig. 13. There is no systematic trend in this phase diagram.

Assuming HD 192639 was a binary system made of two nearly identical stars seen under a low inclination angle, one could expect that the lines of the two stars would remain heavily blended all over the orbital cycle. In this case, the variations of the width of the absorption lines would provide the only "finger-print" of an orbital motion. The broadening of the $\mathrm{H} \beta$ line observed around JD 2451067.4 and JD 2451072.4 yields therefore an upper limit of $\sim 50 \mathrm{~km} \mathrm{~s}^{-1}$ on the radial velocity separation between the two components in a putative binary system around quadrature. However, the fact that the He II and $\mathrm{N}$ III absorption lines (which are expected to be less affected by stellar wind emission and absorption than the $\mathrm{H} \beta$ line) exhibit no trace of a broadening or any other significant variability at the $\nu_{1}$ frequency argues against a binary explanation for the $\nu_{1}$ modulation.

In summary, there is no consistent periodicity in the RVs of the different absorption lines and although we cannot completely rule out a binary system seen under a very low inclination angle, it seems more likely that the line profile variations with the $\nu_{1}$ frequency arise in the stellar wind of a single star.
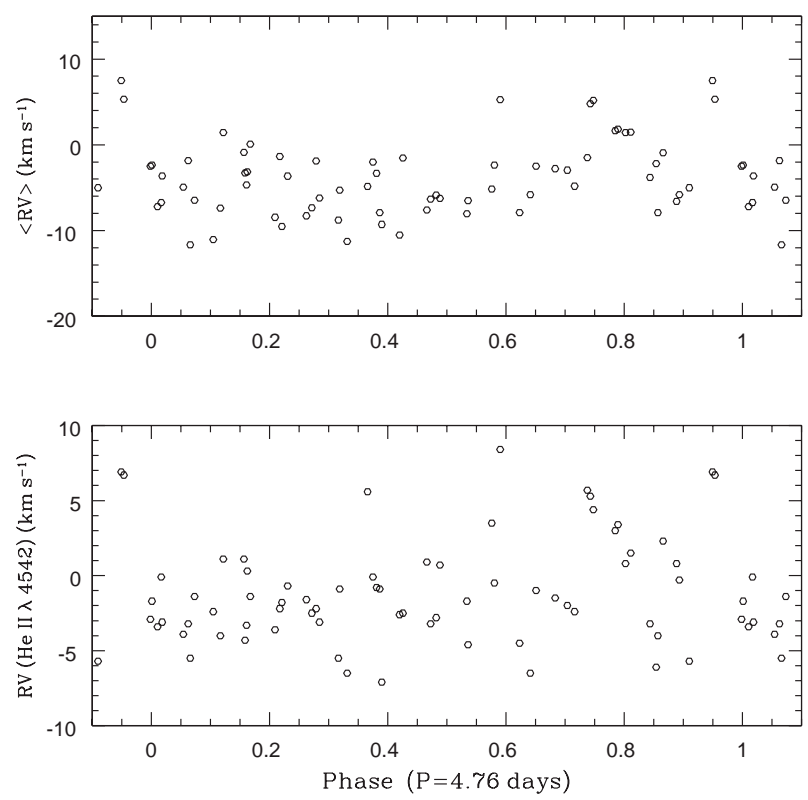

Fig. 13. Upper panel: phase diagram of the mean radial velocity of the He I $\lambda \lambda 4471,4713$ and N III $\lambda \lambda 4511,4515$ absorption lines assuming a period of 4.76 days. Lower panel: same but for the RVs of the He II $\lambda 4542$ line 
We have measured the equivalent widths of the He I $\lambda 4471$ and He II $\lambda 4542$ lines to search for any phasedependence of the intensity ratio of these lines. According to Conti (1973) the ratio $\log W^{\prime}=\log \left(\frac{E W(4471)}{E W(4542)}\right)$ provides an efficient temperature classification criterion for O-stars, provided that both diagnostic lines are formed in the photosphere. Assuming that the structure in the wind of HD 192639 results from some kind of "hot" or "cold spot" at the level of the star's photosphere that covers a significant fraction of the stellar surface, we could in principle set an upper limit on the difference in temperature between this "spot" and the ambient photosphere. We obtain an average value of $\log W^{\prime}=0.045$ for HD 192639 which corresponds to an O7.5 star. The equivalent width ratio displays some variations corresponding to an apparent spectral type between O7.5 and O8. However, these variations are not "phase-locked" with the 4.76-day cycle. Moreover, in the case of HD 192639, the use of the Conti criterion as a diagnostic of the photospheric temperature is probably ambiguous because of the contamination of the photospheric absorption lines by a variable wind emission and/or absorption. Indeed, the variations of the $W^{\prime}$ criterion reflect mainly those of the equivalent width of the He I $\lambda 4471$ line which is most probably partially formed in the wind (see also Paper I). Therefore it seems likely that the variations discussed hereabove do not reflect a genuine change of the local effective temperature but are rather due to a change in the optical depth in the wind.

\section{Discussion}

Our spectroscopic time series reveals the existence of a cyclical modulation of the P-Cygni profiles in the visible spectrum of HD 192639 with a period of $P_{\text {obs }} \simeq$ 4.8 days (assuming that $\nu_{1}$ is indeed the right alias). The phenomenon persisted over at least four years.

Nowadays, the most popular explanation for periodic variability of the stellar wind features in OB-type stars assumes that the observed period corresponds to a meaningful fraction of the rotational period (i.e. $P_{\mathrm{obs}}=P_{\mathrm{rot}} / n$, where $n$ is an integer). Assuming a radius of $19.5 R_{\odot}$ (Herrero et al. 1992; Puls et al. 1996), we can derive an upper limit on $P_{\text {rot. }}$. Using the most recent determinations of the projected rotational velocity $v_{e} \sin i=96 \mathrm{~km} \mathrm{~s}^{-1}$ (Howarth et al. 1997) and $110 \mathrm{~km} \mathrm{~s}^{-1}$ (Penny 1996), we obtain upper limits of 10.30 and 8.99 days respectively. Therefore, in the framework of a rotational modulation hypothesis, the 4.8-day time scale could correspond either to $P_{\text {rot }}$ or $P_{\text {rot }} / 2$. In the former case, the recurrent modulation could be produced by a single feature in the wind, whereas in the latter configuration the lack of a significant signal in the power spectrum near $\nu_{1} / 2$ implies two very similar wind features which would have to be roughly symmetric about the rotation axis. If we assume that $n=1$, we find that $i \sim 30^{\circ} \pm 3^{\circ}$, while $n=2$ yields $i \geq 68^{\circ}$.

\subsection{Comparison with other stars}

The line profile variations detected in the optical wind features of HD 192639 are reminiscent of those detected by Kaper et al. (1997) in the $\mathrm{H} \alpha$ line profile of $\alpha$ Cam (O9.5 Ia) and $\zeta$ Ori (O9.7 Ib). It is worth noting that these authors found that $\mathrm{H} \alpha$ variability is usually concentrated in the low velocity $\left(0.0-0.2 \times v_{\infty}\right)$ part of the line profile. Kaper et al. (1997) also report an episodic strengthening of the emission component in the $\mathrm{H} \alpha$ profile of $19 \mathrm{Cep}$ $(\mathrm{O} 9.5 \mathrm{Ib})$ that is reminiscent of the one we observe in the spectrum of HD 192639 on JD 2451061.66.

So far, the most prominent examples of rotationally modulated winds were found from extensive observations of UV resonance lines with the IUE satellite (see e.g. Howarth et al. 1995; Fullerton et al. 1997; Henrichs et al. 1998; Kaper et al. 1999). In the case of HD 64760 (B0.5 Ib), Fullerton et al. (1997) report a periodic $(P \sim$ 1.2 days) modulation of the mean flux level over most of the P-Cygni absorption trough of the prominent $\mathrm{Si}$ IV, $\mathrm{C}$ IV and N v doublets. An interesting feature of these structures is that a particular phase of the modulation occurs first at an intermediate velocity before it simultaneously reaches higher and lower velocities. This "phase bowing" effect is interpreted by Fullerton et al. (1997) as evidence for a significant azimuthal extent of the structures in the wind of HD 64760. Kaper et al. (1999) report a similar effect for the discrete absorption components (DACs) seen in the resonance lines of $\xi$ Per (O7.5 III). At this point, it is worth noting that the He II $\lambda 4686$ and $\mathrm{H} \alpha$ lines in HD 192639 show no trace whatsoever of such a bowing of the phase constant. Instead, the phase constant $\phi_{0}(v)$ remains roughly constant over the He II $\lambda 4686$ absorption component while it undergoes a systematic decrease towards more negative velocities in the $\mathrm{H} \alpha$ absorption trough. The latter situation is slightly similar to what is seen in the UV resonance lines of most of the O-stars observed by Kaper et al. (1999). As far as the DAC features in the UV lines are concerned, this phase behaviour is usually attributed to an outwards moving accelerating structure.

The difference in phase behaviour between the He II $\lambda 4686$ and $\mathrm{H} \alpha$ lines of HD 192639 is somewhat surprising. The roughly constant $\phi_{0}$ of the He II $\lambda 4686$ absorption component is easily understood in terms of a corotating line forming region confined to the inner region of the wind. On the other hand, the rough similarity between the phase behaviour in the $\mathrm{H} \alpha$ line and typical UV lines (in other stars) points toward a significantly larger radial extent of the $\mathrm{H} \alpha$ line forming region.

The cyclical modulation of the absorption component of the P-Cygni profiles in the visible spectrum of HD 192639 extends to velocities of $\sim-350$ and $-600 \mathrm{~km} \mathrm{~s}^{-1}$ for the He II $\lambda 4686$ and $\mathrm{H} \alpha$ profiles respectively. Assuming that we see the wind modulation head-on and adopting the terminal velocity $v_{\infty}=2150 \mathrm{~km} \mathrm{~s}^{-1}$ determined by Puls et al. (1996), these velocities correspond to $0.16 \times v_{\infty}$ and $0.28 \times v_{\infty}$ respectively. Using the $\beta=0.95$ 
"standard" velocity law of Puls et al. (1996) we find that the variability of the P-Cygni absorptions of the two lines probes the base of the wind out to $1.17 R_{*}$ and $1.35 R_{*}$ respectively. It seems unlikely that such a small difference in the radii of the line forming region could actually account for the different phase behaviour of $\mathrm{H} \alpha$ and He II $\lambda 4686$. It seems therefore likely that the velocity law in the line forming region deviates from a standard law and there could be a velocity plateau associated with the structure that generates the line profile modulation. Such plateaus are predicted for instance in the case of corotating interaction regions (Cranmer \& Owocki 1996).

The most straightforward interpretation of the phase behaviour of the He II $\lambda 4686$ and $\mathrm{H} \alpha$ lines seems to be that the wind rotates roughly as a rigid body out to some distance and then reverts to a Keplerian rotation plus an outwards acceleration due to the radiation pressure. Such a behaviour could be caused by a magnetic field. Indeed, if we adopt the mass loss rate, radius and velocity law derived by Puls et al. (1996) as a first approximation, we find that a bipolar magnetic field of $B_{*} \simeq 115 \mathrm{G}$ yields an Alfvén radius of $1.17 R_{*}$, whilst $B_{*} \simeq 200 \mathrm{G}$ yields an Alfvén radius of $1.35 R_{*}$. Therefore a moderate magnetic field could be sufficient to force the inner wind region, where the bulk of the He II $\lambda 4686$ emission forms, into a rigid body corotation.

\subsection{Towards a possible explanation}

If we accept the idea that the stellar rotation sets the timescale of the variability, we need to imagine a wind geometry that can produce the modulation observed in the spectrum of HD 192639. In this section, we review the pros and cons of two rather simple models that could account for some of the observed properties of the spectral lines discussed hereabove.

Let us first consider the possibility of a structure resulting from a localized "bright spot" on the stellar surface. Such a spot is expected to produce an enhanced mass flux resulting in a localized slow wind stream. Fast wind streams from adjacent regions will collide with the slower wind generating a so-called corotating interaction region (CIR; Cranmer \& Owocki 1996; Owocki 1999). These CIRs are thought to produce the cyclical modulation of the P-Cygni absorptions in UV resonance lines (e.g. Fullerton et al. 1997). The increase of the wind density in the CIR (Cranmer \& Owocki 1996) will allow the $\mathrm{He}$ II $\lambda 4686$ and $\mathrm{H} \alpha$ line formation regions inside the CIR to extend farther away from the star than would be expected for a smooth wind with a $\beta \simeq 1$ standard velocity law. Nevertheless, the formation of the lines probably occurs still rather close to the stellar surface so that the azimuthal extension of the line formation region (at least for He II $\lambda$ 4686) due to the bending of the CIR structure should remain small.

Harries (2000) presented simulations of synthetic line profiles produced by a corotating spiral density enhancement. He finds that this feature generates an S-wave like pattern as a function of rotational phase. The variations of the He II $\lambda 4686$ line displayed in the grey-scale image in Fig. 9 are slightly reminiscent of the synthetic variations simulated by Harries (2000; see his Fig. 4). We note however that the variations observed in the He II $\lambda 4686$ profile of HD 192639 occur over a much smaller velocity range and that the behaviour of the $\mathrm{H} \alpha$ line in the spectrum of HD 192639 is quite different from the predictions of this model. Another important difference concerns the time dependence of the modulation. At a given wavelength, the simulations presented by Harries (2000) yield a rather abrupt and short-lived increase of the intensity, whereas our observations reveal a rather smooth (roughly sinusoidal) variation of the intensity (see our Figs. 8 and 10).

A single CIR would be sufficient to account for the observed modulation of the P-Cygni absorption component. However, if the azimuthal extension of the line formation region in the CIR is indeed small, then a second structure is probably required to account for the simultaneous variability of the emission component. If we assume that these structures arise from diametrically opposite spots on the stellar surface, we are confronted with the problem that the second CIR should essentially be occulted by the stellar core when the first one crosses the line of sight. In this case, we would expect the emission component to be significantly reduced at maximum absorption, which is not the case. The observed variability can thus be explained with a CIR scenario if either the spots that generate the CIRs are not symmetric about the center of the star or the formation region of the He II $\lambda 4686$ and $\mathrm{H} \alpha$ lines extends along the CIRs out to distances where the azimuthal extent becomes quite significant allowing both CIR features to be visible at maximum absorption (see Fig. 14). However, we recall that the phase behaviour of the He II $\lambda 4686$ absorption argues against a noticeable azimuthal bending.

Alternatively, we can imagine a "confined corotating wind" to explain the double-peaked emission component. Conti \& Leep (1974) suggested that the double-peaked He II $\lambda 4686$ emission line profiles observed in Oef stars are produced in a rotating wind. Assuming that such a corotating wind actually exists in the case of HD 192639, the only way to account for the observed modulation of the absorption component is to consider that the rotating wind is confined in latitude and that its symmetry axis is tilted with respect to the rotation axis. A situation like this could result for instance if HD 192639 were an oblique magnetic rotator. Such a model has been suggested to explain the line profile variability observed in the spectra of $\zeta$ Pup (Moffat \& Michaud 1981) and $\theta^{1}$ Ori C (Stahl et al. 1996). Babel \& Montmerle (1997) found that a strong enough magnetic field $\left(B_{*} \simeq 270-370 \mathrm{G}\right.$ ) can indeed confine a significant fraction of the wind of $\theta^{1}$ Ori $\mathrm{C}$ into a "circumstellar cooling disk" located in the plane of the magnetic equator. The variations of the absorption component of the He II $\lambda 4686$ line in HD 192639 (Fig. 9) are indeed a bit reminiscent of the behaviour of this line in $\theta^{1}$ Ori C (Stahl et al. 1996). The amplitude of the 


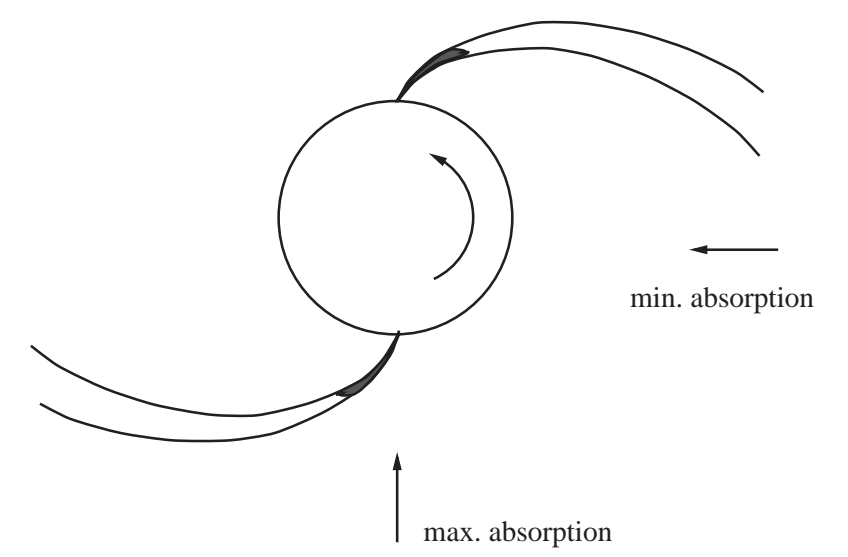

Fig. 14. Schematic view of a CIR structure in HD 192639. The star is shown pole-on and the lines of sight are projected on the equatorial plane. The line formation region along the CIRs is shown by the dark regions. In this cartoon, we have arbitrarily assumed that the spots that generate the CIR are located on the stellar equator. If the spots are diametrically opposite, we find that for reasonable values of the inclination of the rotation axis (and the latitude of the spots), the second CIR should be occulted by the stellar core when the first one passes in front of the star (i.e. at maximum absorption strength)

variations in $\theta^{1}$ Ori $\mathrm{C}$ is however about a factor of 10 larger than in HD 192639. Another difference between the two stars is that the lines in the optical spectrum of $\theta^{1}$ Ori $\mathrm{C}$ do not display a P-Cygni type profile.

Let us assume that the line-forming region is a kind of corotating torus tilted with respect to the rotation axis (see Fig. 15). In this configuration, we expect a priori to observe a double-peaked emission line morphology at all rotational phases. Assuming a solid-body rotation law (star + torus), the separation between the two emission peaks would vary roughly between $2 v_{\text {rot }} \sin i$ and $2 v_{\text {rot }} \sin i \cos \theta$ where $v_{\text {rot }}$ stands for the rotation velocity of the torus at the intersection between the torus and the stellar (rotational) equator and where $\theta$ is the tilt angle of the torus (i.e. the angle between the symmetry axis of the torus and the rotation axis). Of course, the strongest P-Cygni absorption is expected when the torus crosses the line of sight. If $i \sim 30^{\circ}$ (as expected for $P_{\text {obs }}=P_{\text {rot }}$ ), then maximum absorption is predicted to occur when the separation between the two emission peaks is maximum. One would therefore expect to observe a well defined doublepeaked emission component around maximum absorption, which is not the case. If instead $i \geq 68^{\circ}$, then the overall picture depends on the value of $\theta$ and maximum absorption can actually occur when the separation between the two peaks is about minimum whilst minimum absorption happens when the two peaks are clearly separated. For reasonable values of the tilt angle $\theta$, we can expect that the separation between the peaks near maximum absorption becomes so small that the peaks are no longer seen as two individual features. Adopting an inclination above $68^{\circ}$ and assuming that the wind is confined into a tilted torus allows thus to account qualitatively for most of the observational properties of the P-Cygni profiles in the visible spectrum of HD 192639.

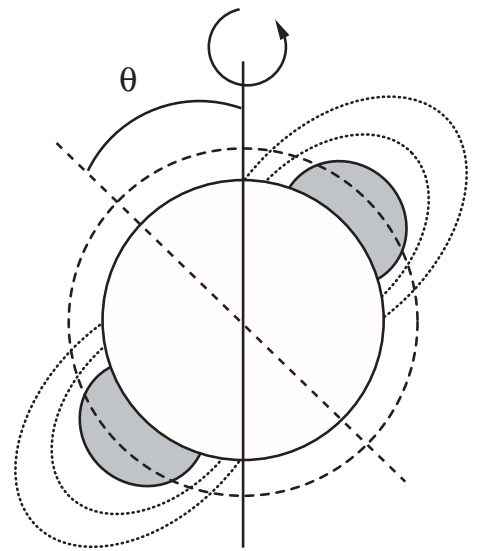

Fig. 15. Schematic view of the confined corotating wind model. The cartoon shows a cross-section across the star by the plane defined by the rotation axis and the magnetic axis. The dashed line indicates the magnetic axis tilted by an angle $\theta$ with respect to the rotation axis. The dashed circle corresponds to the Alfvén radius. The confined wind region is schematically represented by the dotted surfaces. The line formation region in the confined wind is shown by the hatched regions

\section{Concluding remarks and future work}

In this paper, we have shown that the absorption components of the P-Cygni profiles in the optical spectrum of HD 192639 display a periodic modulation with a period of roughly 4.8 days. This period is also found in the emission component of the profiles though with a significantly lower amplitude.

Although we cannot completely rule out a binary system seen under a very low inclination angle, the most likely explanation for the 4.8-day clock is that this period reflects the rotational period (or a meaningful fraction of it) of a single star. Beside the variability of the profile, further evidence for a non-spherical stellar wind comes from the double-peaked structure of the He II $\lambda 4686$ and (to some extent) $\mathrm{H} \alpha$ lines. We have discussed two rather simple geometries for the stellar wind in HD 192639. Both a corotating interaction region produced by a local enhancement of the mass loss or a tilted confined corotating wind can be in qualitative agreement with the most important observational properties of the spectral lines discussed in this paper. Detailed simulations of the line profiles adopting the geometries discussed above are needed to further constrain the origin of the phenomenon and to estimate its impact on the accuracy of the mass-loss rates derived from standard model atmosphere codes (Puls et al. 1996). Such quantitative simulations are beyond the scope of the present paper.

If we accept the idea of rotational modulation, we still need to specify the mechanism that generates the structure (be it a CIR or a tilted confined corotating wind) responsible for the observed variation. A magnetic field anchored in the star offers an attractive way to produce such a structure. However, as stated in the introduction, so far there has been no direct detection of such 
a magnetic field in a massive star. On the other hand, we can look for other indirect evidence. For instance, it has been suggested that the non-thermal radio emission observed in some OB stars is due to synchrotron radiation of relativistic electrons in the stellar winds (Chen \& White 1994 and references therein). Unfortunately, HD 192639 is too faint to be detected in the radio domain and only an upper limit on the flux density at $8.45 \mathrm{GHz}$ is available (Scuderi et al. 1998). Another, maybe more promising, way to learn more about the nature of this intriguing star could be through phase-resolved high resolution spectropolarimetric observations. Finally, high spectral resolution $(\geq 50000)$, high signal to noise and high time resolution spectroscopy of a "wind-free" photospheric absorption line such as He II $\lambda 4542$ would allow to search for non-radial pulsations that might play a key role in generating the wind variability.

Acknowledgements. We wish to express our thanks to Tracy L. Smith and David C. Knauth for taking some of the Ritter spectra and to Asif ud-Doula for helpful discussions. We are also grateful to the referee Dr. Alex W. Fullerton for his helpful report. GR would like to thank the staff of the Observatoire de Haute Provence for their technical support during the various observing runs. GR, JMV and EG are greatly indebted to the Fonds National de la Recherche Scientifique (Belgium) for multiple assistance including the financial support for the rent of the OHP telescope in 1999 through contract 1.5.051.00 "Crédit aux Chercheurs" FNRS. This research is also supported in part by contract P4/05 "Pôle d'Attraction Interuniversitaire" (SSTC-Belgium). Partial support through the PRODEX XMM-OM Project is also gratefully acknowledged. The travels to OHP for the observing runs were supported by the Ministère de l'Enseignement Supérieur et de la Recherche de la Communauté Française. The SIMBAD database has been consulted for the bibliography.

\section{References}

Abt, H. A., \& Morrell, N. I. 1995, ApJS, 99, 135

Babel, J., \& Montmerle, T. 1997, ApJ, 485, L29
Chen, W., \& White, R. L. 1994, Ap\&SS, 221, 259

Conti, P. S. 1973, ApJ, 179, 181

Conti, P. S., \& Leep, E. M. 1974, ApJ, 193, 113

Cranmer, S. R., \& Owocki, S. P. 1996, ApJ, 462, 469

de Jong, J. A., Henrichs, H. F., Schrijvers, C., et al. 1999, A\&A, 345,172

Fullerton, A. W. 1999, in IAU Coll. 169, Variable and Nonspherical Stellar Winds in Luminous Hot Stars, ed. B. Wolf, O. Stahl, \& A. W. Fullerton, Lect. Notes Phys., 523, 3

Fullerton, A. W., Gies, D. R., \& Bolton, C. T. 1996, ApJS, 103,475

Fullerton, A. W., Massa, D. L., Prinja, R. K., Owocki, S. P., \& Cranmer, S. R. 1997, A\&A, 327, 699

Harries, T. J. 2000, MNRAS, 315, 722

Heck, A., Manfroid, J., \& Mersch, G. 1985, A\&AS, 59, 63

Henrichs, H. F., de Jong, J. A., Nichols, J. S., et al. 1998, in Ultraviolet Astrophysics Beyond the IUE Final Archive, ESA-SP 413, 157

Herrero, A., Kudritzki, R. P., Vilchez, J. M., et al. 1992, A\&A, 261,209

Howarth, I. D., Prinja, R. K., \& Massa, D. 1995, ApJ, 452, L65

Howarth, I. D., Siebert, K. W., Hussain, G. A. J., \& Prinja, R. K. 1997, MNRAS, 284, 265

Kaper, L., Henrichs, H. F., Fullerton, A. W., et al. 1997, A\&A, 327,281

Kaper, L., Henrichs, H. F., Nichols, J. S., \& Telting, J. H. 1999, A\&A, 344, 231

Massa, D., Fullerton, A. W., Nichols, J. S., et al. 1995, ApJ, 452, L53

Mathys, G. 1999, in IAU Coll. 169, Variable and Non-spherical Stellar Winds in Luminous Hot Stars, ed. B. Wolf, O. Stahl, \& A. W. Fullerton, Lect. Notes Phys., 523, 95

Moffat, A. F. J., \& Michaud, G. 1981, ApJ, 251, 133

Owocki, S. P. 1999, in IAU Coll. 169, Variable and Nonspherical Stellar Winds in Luminous Hot Stars, ed. B. Wolf, O. Stahl, \& A. W. Fullerton, Lect. Notes Phys., 523, 294

Penny, L. R. 1996, ApJ, 463, 737

Puls, J., Kudritzki, R.-P., Herrero, A., et al. 1996, A\&A, 305, 171

Rauw, G., \& Vreux, J.-M. 1998, A\&A, 335, 995, Paper I

Scuderi, S., Panagia, N., Stanghellini, C., Trigilio, C., \& Umana, G. 1998, A\&A, 332, 251

Stahl, O., Kaufer, A., Rivinius, T., et al. 1996, A\&A, 312, 539 